\title{
Expansion Maps In D-Metric And Tri D-Metric Spaces
}

\author{
A.S.Saluja and Alkesh Kumar Dhakde \\ Deptt. Of Mathematics, J.H.Govt. P.G. College, Betul (M.P.), India \\ IES College of Technology, Bhopal (M.P.), India
}

ABSTRACT In this paper, we obtain some results on fixed points for expansion mappings in D-metric and Tri D-metric spaces, introduced by Dhage [1] .Our results includes several fixed point results in ordinary metric spaces as special cases on the line of Maia [5].

KEYWORDS AND PHRASES: Fixed point, D-metric spaces, Expansion maps, etc.

SUBJECT CLASSIFICATION: Primary 47H10, Secondary $54 \mathrm{H} 25$.

\section{INTRODUCTION:}

Motivated by the measure of nearness, the concept of a D-metric space introduced by Dhage [1] is as follows:

A nonempty set $X$ together with a function $\rho: X \times X \times X \rightarrow[0, \infty)$, is called a Dmetric space with a D-metric $\rho$, denoted by $(X, \rho)$, if $\rho$ satisfies the following properties:

(i) $\rho(x, y, z)=0 \Leftrightarrow x=y=z$ (Coincidence) for all $x, y, z \in X$

(ii) $\rho(x, y, z)=\rho(\rho\{x, y, z\})$ (Symmetry) Where $\rho$ is a permutation function.

(iii) $\rho(x, y, z) \leq \rho(x, y, a)+\rho(x, a, z)+\rho(a, y, z)$ for all $x, y, z, a \in X$. (Tetrahedral inequality)

A sequence $\left\{x_{n}\right\} \subset X$, is said to be D-converges to a point $x \in X$ if $\lim _{m, n \rightarrow \infty} \rho\left(x_{m}, x_{n}, x\right)=0$. Similarly, a sequence $\left\{x_{n}\right\} \subset X$, is called D-Cauchy if $\lim _{m, n, p \rightarrow \infty} \rho\left(x_{m}, x_{n}, x_{p}\right)=0$. A complete D-metric space is one in which every D-Cauchy sequence converges to a point in it. A subset $\mathrm{S}$ of a D-metric space $X$ is called bounded, if there exists a constant $K>0$, such that $\rho(x, y, z) \leq K$ for all $x, y, z \in S$. The infimum of all such $k$ is called the diameter of $S$ and is denoted by $\delta(S)$.

Let $f: X \rightarrow X$, then the orbit of $f$ at a point $x \in X$ is a set in $X$, defined by $O_{f}(x)=\left\{x, f x, f^{2} x, \ldots.\right\}$. Again a D-metric space is called f-orbitally bounded if there exists a constant $M>0$ such that $\rho(x, y, z) \leq M$ for all $x, y, z \in O_{f}(x)$.A D-metric space is called forbitally complete if every D-Cauchy sequence in $O_{f}(x)$ converges to a point in $X$.

It is known that the D-metric $\rho$ is a continuous function on $X^{3}$ in the topology of Dmetric convergence which is Hausdorff, see Dhage [2].

In 1976, Rosenholtz [7] discussed local expansion mappings. Let $(X, d)$ be an ordinary metric space. Then a mapping $T: X \rightarrow X$, expansive on a subset $B$ of $X$, if $d(T x, T y)>d(x, y)$ for all $x, y \in B$ with $x \neq y$.

$T$ is a Local expansion if every point in $T$ has a neighbourhood $B$ on which $T$ is expansive.

In fact Rosenholtz proved, "If $(X, d)$ be a complete metric space and $T: X \rightarrow X$ be a self map of $X$ onto itself satisfying; $d(T x, T y)>\lambda d(x, y)$ for all $x, y \in X$ with $x \neq y$ and $\lambda>1$. Then $T$ has a fixed point in $X "$

We need the following D-Cauchy principle developed by Dhage [3]. 
Lemma 1: (D-Cauchy principle): Let $\left\{x_{n}\right\}$ be bounded sequence with D-bound $K$, satisfying:

(1.1.1) $\rho\left(y_{n}, y_{n+1}, y_{p}\right) \leq \lambda^{n} K$, for all $n, p \in N$ with $p>n$, where $0 \leq \lambda<1$. Then $\left\{y_{n}\right\}$ is a D-Cauchy sequence.

\section{Throughout in this paper we use the symbol}

$$
\rho(x, y, z) \cdot \rho(x, y, z)=\{\rho(x, y, z)\}^{2}=\rho^{2}(x, y, z)
$$

\section{MAIN RESULTS:}

THEOREM 2.1 : Let $f: X \rightarrow X$ be a surjective mapping of a f-orbitally bounded and forbitally complete $\mathrm{D}$-metric space $(X, \rho)$.If there exists non-negative reals $a_{1}, a_{2}, \ldots . a_{7}$ with $a_{1}+a_{3}+a_{5}>0, a_{2}<1$ and $a_{1}+a_{2}+a_{3}+a_{4}+a_{5}+a_{6}+a_{7}>1$, such that ;

$$
\begin{aligned}
\rho^{2}(f x, f y, f z) & \geq a_{1} \cdot \rho^{2}(x, y, z)+a_{2} \cdot \rho^{2}(x, f x, f z)+a_{3} . \rho^{2}(y, f y, z)+a_{4} . \rho(x, f x, f z) \rho(x, y, z) \\
& +a_{5} . \rho(y, f y, z) \rho(x, y, z)+a_{6} . \rho(x, f x, f z) \rho(y, f y, z)+a_{7} . \rho(f x, f y, f z) \rho(x, y, z)
\end{aligned}
$$

for all $x, y, z \in X$ with $x \neq y \neq z$. Then $f$ has a fixed point in $X$.

PROOF: Let $x_{0} \in X$. Since $\mathrm{f}$ is surjective, there exists an element $x_{1}$ satisfying $x_{1} \in f^{-1}\left(x_{0}\right)$.By the way we can take $x_{n} \in f^{-1}\left(x_{n-1}\right), n=2,3,4, \ldots \ldots \ldots$.

If $x_{m}=x_{m-1}$ for some $m$, then $x_{m}$ is a fixed point of $f$.

Without loss of generality, we can assume $x_{n} \neq x_{n-1}$ for every $n$.From (2.1.1), we have

$$
\begin{aligned}
\rho^{2}\left(x_{n-1}, x_{n}, x_{n+p-1}\right) & =\rho^{2}\left(f x_{n}, f x_{n+1}, f x_{n+p}\right) \\
& \geq a_{1} \cdot \rho^{2}\left(x_{n}, x_{n+1}, x_{n+p}\right)+a_{2} \cdot \rho^{2}\left(x_{n}, f x_{n}, f x_{n+p}\right)+a_{3} \cdot \rho^{2}\left(x_{n+1}, f x_{n+1}, x_{n+p}\right) \\
& +a_{4} \cdot \rho\left(x_{n}, f x_{n}, f x_{n+p}\right) \cdot \rho\left(x_{n}, x_{n+1}, x_{n+p}\right)+a_{5} \cdot \rho\left(x_{n+1}, f x_{n+1}, x_{n+p}\right) \cdot \rho\left(x_{n}, x_{n+1}, x_{n+p}\right) \\
& +a_{6} \cdot \rho\left(x_{6}, f x_{n}, f x_{n+p}\right) \cdot \rho\left(x_{n+1}, f x_{n+1}, x_{n+p}\right)+a_{7} \cdot \rho\left(f x_{n}, f x_{n+1}, f x_{n+p}\right) \cdot \rho\left(x_{n}, x_{n+1}, x_{n+p}\right) \\
& =a_{1} \cdot \rho^{2}\left(x_{n}, x_{n+1}, x_{n+p}\right)+a_{2} \cdot \rho^{2}\left(x_{n}, x_{n-1}, x_{n+p-1}\right)+a_{3} \cdot \rho^{2}\left(x_{n+1}, x_{n}, x_{n+p}\right) \\
& +a_{4} \cdot \rho\left(x_{n}, x_{n-1}, x_{n+p-1}\right) \cdot \rho\left(x_{n}, x_{n+1}, x_{n+p}\right)+a_{5} \cdot \rho\left(x_{n+1}, x_{n}, x_{n+p}\right) \cdot \rho\left(x_{n}, x_{n+1}, x_{n+p}\right) \\
& +a_{6} \cdot \rho\left(x_{n}, x_{n-1}, x_{n+p-1}\right) \cdot \rho\left(x_{n+1}, x_{n}, x_{n+p}\right)+a_{7} \cdot \rho\left(x_{n-1}, x_{n}, x_{n+p-1}\right) \cdot \rho\left(x_{n}, x_{n+1}, x_{n+p}\right)
\end{aligned}
$$

Thus,

$$
\begin{gathered}
\left(a_{1}+a_{3}+a_{5}\right) \cdot \rho^{2}\left(x_{n}, x_{n+1}, x_{n+p}\right)+\left(a_{4}+a_{6}+a_{7}\right) \cdot \rho\left(x_{n-1}, x_{n}, x_{n+p-1}\right) \cdot \rho\left(x_{n}, x_{n+1}, x_{n+p}\right) \\
-\left(1-a_{2}\right) \cdot \rho^{2}\left(x_{n-1}, x_{n}, x_{n+p}\right) \leq 0
\end{gathered}
$$

Or,

(2.1.2) $\quad\left(a_{1}+a_{3}+a_{5}\right) t^{2}+\left(a_{4}+a_{6}+a_{7}\right) t-\left(1-a_{2}\right) \leq 0$, where

(2.1.3) $t=\left\lfloor\rho\left(x_{n}, x_{n+1}, x_{n+p}\right) / \rho\left(x_{n-1}, x_{n}, x_{n+p-1}\right)\right\rfloor$

Let $g:[0, \infty) \rightarrow R$ be the function

(2.1.4) $g(t)=\left(a_{1}+a_{3}+a_{5}\right) t^{2}+\left(a_{4}+a_{6}+a_{7}\right) t-\left(1-a_{2}\right)$

Then from the hypothesis, $g(0)=a_{2}-1<0$

and $g(1)=a_{1}+a_{2}+a_{3}+a_{4}+a_{5}+a_{6}+a_{7}-1>0$. 
Let $k \in(0,1)$ be the root of the equation $g(t)=0$.

Then, $g(t) \leq 0$ for $t \leq k$ and therefore

$$
\begin{aligned}
\rho\left(x_{n}, x_{n+1}, x_{n+p}\right) & \leq k . \rho\left(x_{n-1}, x_{n}, x_{n+p-1}\right) \\
& \leq \mathrm{k}^{2} . \rho\left(x_{x-2}, x_{n-1}, x_{n+p-2}\right) \\
& \leq \ldots \ldots \ldots \ldots \ldots \ldots \ldots \ldots \ldots \ldots \\
& \leq k^{n} M
\end{aligned}
$$

Where $\mathrm{M}$ is a D-bound of $0_{f}(x)$.

Now, an application of Lemma 2.1 yields that $\left\{x_{n}\right\}$ is a D-Cauchy sequence. Since $X$ is f-orbitally complete, there is a point $x \in X$ such that, $\lim _{n \rightarrow \infty} x_{n}=x$.

Now, we shall show that $x$ is a fixed point of $f$.

Since, $f$ is surjective there exists $y$ in $X$, such that $y \in f^{-1}(x)$

For infinitely many $n, x_{n} \neq x$, hence for such $n$, we have

$$
\begin{aligned}
\rho^{2}\left(x_{n}, x, x\right) & =\rho^{2}\left(f x_{n+1}, f y, f y\right) \\
& \geq a_{1} \cdot \rho^{2}\left(x_{n+1}, y, y\right)+a_{2} \cdot \rho^{2}\left(x_{n+1}, f x_{n+1}, f y\right)+a_{3} \cdot \rho^{2}(y, f y, y) \\
& +a_{4} \cdot \rho\left(x_{n+1}, f x_{n+1}, f y\right) \cdot \rho\left(x_{n+1}, y, y\right)+a_{5} \cdot \rho(y, f y, y) \cdot \rho\left(x_{n+1}, y, y\right) \\
& +a_{6} \cdot \rho\left(x_{n+1}, f x_{n+1}, f y\right) \cdot \rho(y, f y, y)+a_{7} \cdot \rho\left(f x_{n+1}, f y, f y\right) \cdot \rho\left(x_{n+1}, y, y\right) \\
& =a_{1} \cdot \rho^{2}\left(x_{n+1}, y, y\right)+a_{2} \cdot \rho^{2}\left(x_{n+1}, x_{n}, x\right)+a_{3} \cdot \rho^{2}(y, x, y) \\
& +a_{4} \cdot \rho\left(x_{n+1}, x_{n}, x\right) \cdot \rho\left(x_{n+1}, y, y\right)+a_{5} \cdot \rho(y, x, y) \cdot \rho\left(x_{n+1}, y, y\right) \\
& +a_{6} \cdot \rho\left(x_{n+1}, x_{n}, x\right) \cdot \rho(y, x, y)+a_{7} \cdot \rho\left(x_{n}, x, x\right) \cdot \rho\left(x_{n+1}, y, y\right)
\end{aligned}
$$

On letting $n \rightarrow \infty$, we obtain

$$
\begin{aligned}
0 & \geq a_{1} \cdot \rho^{2}(x, y, y)+a_{2} \cdot \rho^{2}(x, x, x)+a_{3} \cdot \rho^{2}(y, x, y)+a_{4} \cdot \rho(x, x, x) \cdot \rho(x, y, y) \\
& +a_{5} \cdot \rho(y, x, y) \cdot \rho(x, y, y)+a_{6} \cdot \rho(x, x, x) \cdot \rho(y, x, y)+a_{7} \cdot \rho(x, x, x) \cdot \rho(x, y, y) \\
& =\left(a_{1}+a_{3}+a_{5}\right) \cdot \rho^{2}(x, y, y)
\end{aligned}
$$

Since, $a_{1}+a_{3}+a_{5}>0$, So $x=y$.

Thus $x$ is a fixed point of $f$.

This completes the proof.

COROLLARY 2.2: Let $f: X \rightarrow X$ be a surjective mapping of a f-orbitally bounded and forbitally complete D-metric space $X$.If there exists a real constant $k>1$, such that

(2.2.1) $\quad \rho^{2}(f x, f y, f z) \geq k . \rho^{2}(x, y, z)$ for all $x, y, z \in X$ with $x \neq y \neq z$.

Then $f$ has a fixed point in $X$.

PROOF : Proof of the corollary 2.2 follows easily from theorem 2.1.

3. It is possible that a D-metric space which is complete w.r.t. a D-metric but may not be complete w.r.t. another D-metric on $X$. In this section we consider a D-metric space with three D-metrics, i.e. a tri D-metric space and investigate some results on the fixed points on the line of Maia [5].

THEOREM 3.1: Let $X$ be a D-metric space with three D-metrics $\rho, \rho_{1}$ and $\rho_{2}$. Let $f: X \rightarrow X$ be a surjective mapping. If there exists non-negative reals $a_{1}, a_{2}, a_{3}, \ldots . . a_{7}$ with $a_{1}+a_{3}+a_{5}>0, a_{2}<1$ and $a_{1}+a_{2}+a_{3}+a_{4}+a_{5}+a_{6}+a_{7}>1$, such that the following conditions hold in $X$; 
(i) $\quad \rho_{2}(x, y, z) \leq \rho_{1}(x, y, z) \leq \rho(x, y, z)$ for all $x, y, z \in X$.

(ii) $\quad X$ is f-orbitally bounded and f-orbitally complete w.r.t. $\rho_{1}$

(iii) $\quad f$ is continuous w.r.t. $\rho_{2}$.

(iv) $\quad f$ Satisfies condition (2.1.1) w.r.t. $\rho$.

Then $f$ has a fixed point in $X$.

PROOF: Let $x_{0} \in X$. Since $f$ is surjective, there exists an element $x_{1}$ satisfying $x_{1} \in f^{-1}\left(x_{0}\right)$.By the same way we can take

$$
x_{n} \in f^{-1}\left(x_{n-1}\right), n=2,3,4, \ldots \ldots \ldots
$$

Then proceeding as in the proof of theorem (2.1), with similar arguments, we get

$$
\rho\left(x_{n}, x_{n+1}, x_{n+p}\right) \leq k^{n} \rho\left(x_{0}, x_{1}, x_{p}\right)
$$

Since, $\rho_{1} \leq \rho$ on $X^{3}$, we have

$$
\begin{aligned}
\rho_{1}\left(x_{n}, x_{n+1}, x_{n+p}\right) & \leq \rho\left(x_{n}, x_{n+1}, x_{n+p}\right) \\
& \leq \mathrm{k}^{\mathrm{n}} \rho\left(x_{0}, x_{1}, x_{p}\right) \\
& \leq k^{n} M, \text { where } \mathrm{M} \text { is a D - bound of } 0_{f}(x) \text { w.r.t. } \rho_{1}
\end{aligned}
$$

Now, an application of Lemma 2.1 yields that $\left\{x_{n}\right\}$ is a D-Cauchy sequence in $X$.

w.r.t. $\rho_{1}$. Since $X$ is f-orbitally complete w.r.t. $\rho_{1}$, there exists a point $x \in X$ such that,

$$
\lim _{n \rightarrow \infty} x_{n}=x
$$

Again since, $\rho_{2} \leq \rho_{1}$ on $X^{3}$, we have

$$
\begin{aligned}
& \lim _{n \rightarrow \infty} \rho_{2}{ }^{2}\left(x_{n}, x, x\right) \leq \lim _{n \rightarrow \infty} \rho_{1}^{2}\left(x_{n}, x, x\right)=0 \\
& \text { Or }, \lim _{n \rightarrow \infty} \rho_{2}{ }^{2}\left(x_{n}, x, x\right)=0
\end{aligned}
$$

This implies that the sequence $\left\{x_{n}\right\}$ converges to $x$ w.r.t. $\rho_{2}$.

Now, by the continuity of $f$ w.r.t. $\rho_{2}$ it follows that

$$
x=\lim _{n \rightarrow \infty} x_{n}=\lim _{n \rightarrow \infty} f x_{n+1}=f\left[\lim _{n \rightarrow \infty} x_{n}\right]=f x
$$

Thus $x$ is a fixed point of $f$. This completes the proof.

COROLLARY 3.2: Let $X$ be a D-metric space with three D-metrics $\rho, \rho_{1}$ and $\rho_{2}$.Let $f: X \rightarrow X$ be a surjective mapping. If there exists a real constant $k>1$, such that, the following conditions hold in $X$;

(i) $\quad \rho_{2}(x, y, z) \leq \rho_{1}(x, y, z) \leq \rho(x, y, z)$ for all $x, y, z \in X$

(ii) $\quad X$ is f-orbitally bounded and f-orbitally complete w.r.t. $\rho_{1}$

(iii) $\quad f$ is continuous w.r.t. $\rho_{2}$.

(iv) $\quad f$ Satisfies condition (2.2.1) w.r.t. $\rho$.

Then $f$ has a fixed point in $X$.

PROOF: Proof of the corollary 3.2 follows easily from theorem 3.1.

\section{REFERENCES:}

\section{DHAGE, B.C.,}

[1] Generalized metric spaces and mapping with fixed point, Bull. Calcutta Math. Soc., 84 (1992), 329-336.

[2] Generalized metric space and topological structure I, Analene, Stint, Univ. "AL. I. Cuza”, Iasi 45 (1999). 
[3] On Kannan type maps in D-metric spaces, Jour. Nat. and Phy. Sci. II (1997).

DHAGE, B.C. AND RHOADES, B.E.

[4] A comparison of two contraction principles, Math. Sci. Res., Hot-Line, 3 (8), (1999), 4953.

MAIA, M.G.

[5] Un osscravazions sulle contrazoni metriche, Rend. Sem. Math. Padova, 40 (1968), 139143.

\section{RHOADES, B.E.}

[6] A comparison of various definitions of contractive mappings, Trans. Amer. Math. Soc., (1976), 257-290.

ROSENHOLTZ;

[7] Local expansion derivatives and fixed points, Fund. Math., 91 (1976), 1-4.

WONG, S.Z., LI., B.Y., GAO, Z.M., ISEKI, K.;

[8] Some fixed point theorems on expansion mappings, Math. Japonica 29, 4(1984), 631-636. 